\title{
La Reserva Forestal El Montoso y el registro de la esperanza Anaphidna Gorochov \& Cadena-Castañeda 2012 (Orthoptera: Tettigoniidae) en Panamá
}

\author{
Alonso Santos Murgas ${ }^{1,2}$ (D) Roberto Cambra1 ${ }^{\text {(D) }}$ \& Carlos A. Gómez González ${ }^{1,3,4}$ \\ 1Universidad de Panamá, Facultad de Ciencias Naturales Exactas y Tecnología, Museo de Invertebrados G. B. \\ Fairchild. Email: alonso.santos@up.ac.pa; cambramiup60@gmail.com; \\ ${ }^{2}$ Sociedad Mesoamericana Para La Biología y la Conservación-Capítulo de Panamá. \\ ${ }^{3}$ Asociación de Estudiantes de Biología, Universidad de Panamá. \\ ${ }^{4}$ Colección de Aves del Instituto Smithsonian, Panamá.
}

Fecha de Recepción: 11-III-2020. Fecha de Aceptación: 23-V-2020. Fecha de Publicación: 25-VI-2020.

\section{RESUMEN}

La Reserva Forestal El Montoso son pequeños reductos de bosque maduros, que aún se conservan casi intactos y que se ven seriamente amenazados por la ampliación de la zona agrícola. Cabe destacar que se registra por primera vez para Panamá y en esta Reserva Forestal El Montoso, el insecto Anaphidna sp. prob. hernandezi Cadena-Castañeda 2012 (Orthoptera: Tettigoniidae), el cual solo se tenía registro para Costa Rica. Se mencionan datos de su distribución geográfica actualizada y sobre su ecología.

Palabras Clave: Nuevo reporte, saltamontes, Reserva Forestal El Montoso

\section{The Forest Reserve of El Montoso and new record of the Katydids Anaphidna} Gorochov \& Cadena Castañeda, 2012 (Orthoptera: Tettigoniidae) in Panama

\section{ABSTRACT}

El Montoso Forest Reserve are small, mature forest redoubts, which are still almost intact and are seriously threatened by the expansion of the agricultural area. It should be noted that the Anaphidna sp. prob. hernandezi Cadena-Castañeda 2012 (Orthoptera: Tettigoniidae), which was only registered in Costa Rica, is registered for the first time in Panama and in this El Montoso Forest Reserve. Data on its updated geographical distribution and on its ecology are mentioned.

Keywords: New record, katydids, the Montoso Forest Reserve

\section{INTRODUCCIÓN}

Las esperanzas o Tettigoniidae (Orthoptera: Ensifera) contiene más de 7500 especies en 22 subfamilias existentes, 96 tribus y 1277 géneros (Eades \& Otte, 2016). En Panamá se reconocen 85 géneros y unas 163 especies bien reconocidas (Nickle, 1992; Santos et al., 2017). Parte del suborden Ensifera, Tettigoniidae es la única familia en la superfamilia Tettigonioidea. Los tettigonidos varían en tamaño desde tan pequeño como $5 \mathrm{~mm}$ a tan grande como $130 \mathrm{~mm}$. Los insectos de la familia Tettigoniidae son comúnmente llamados grillos de arbusto, katydids o saltamontes u esperanzas en América. 
Es importante mencionar algunas características de los Tettigoniidae, muchas especies, principalmente las más pequeñas típicamente viven en hábitats más secos, que pueden inducir a su pequeño tamaño (Rentz, 2010). El pequeño tamaño se asocia con mayor agilidad, desarrollo más rápido y menores necesidades nutricionales. Los tettigoniidos son insectos de los árboles que se oyen más comúnmente durante la noche durante el verano y principios de la época lluviosa en los trópicos. Una de las diferencias más visible de los tettigonidos y los saltamontes, es por la longitud de sus antenas filamentosas, que pueden exceder su propia longitud corporal, mientras que las antenas de saltamontes son siempre relativamente cortas y espesadas (Rentz, 2010).

La actividad de este grupo de insectos es principalmente nocturna, con ruidos estridentes, para llamadas de apareamiento, varias especies exhiben mimetismo y camuflaje, comúnmente con formas y colores similares a las formas de hojas. Los tettigonidos generalmente su ciclo de vida es de aproximadamente un año, por lo general llegan a adulto al final de su ciclo. Las hembras suelen poner sus huevos al final de la época seca, bajo el suelo o en los agujeros del tallo de las plantas. Los huevos son típicamente de forma ovalada y colocados en filas en la planta huésped.

La forma de su ovipositor se relaciona con su adaptabilidad funcional en las áreas donde pone huevos. Consiste en un máximo de tres pares de apéndices formados para conducir el huevo, preparar el lugar donde pondrá sus huevos y colocarlo adecuadamente. Los tettigonidos tienen ovipositores en forma de hoz. Cuando eclosionan los tettigonidos de los huevos, las ninfas a menudo parecen versiones más pequeñas de los adultos, pero en algunas especies, las ninfas no se parecen nada a los adultos y más bien imitan a otras especies como las arañas y los insectos peligrosos u a flores, para prevenir la depredación. Las ninfas permanecen en un estado mímico sólo hasta que son lo suficientemente grandes como para escapar de la depredación. Una vez que terminan su última muda, entonces se preparan para aparearse (Rentz, 2010).

Las esperanzas u tettigonidos se encuentran en todos los continentes, excepto en la Antártida. La gran mayoría de especies de esperanzas viven en las regiones tropicales del mundo. Por ejemplo, la selva tropical de la cuenca del Amazonas es el hogar de más de 2000 especies de esperanzas. Sin embargo, las esperanzas se encuentran en las regiones frías, templadas secas, también, con cerca de 255 especies en Norteamérica (Roberson, 2010).

La alimentación de los tettigonidos incluye hojas, flores, corteza y semillas, pero muchas especies son exclusivamente depredadoras, alimentándose de otros insectos, caracoles o incluso de pequeños vertebrados como serpientes y lagartos. Algunas también son consideradas como plagas por los cultivadores de cultivos comerciales, pero las densidades de población son generalmente bajas, por lo que el causar un gran impacto económico es muy raro (Gwynne \& William, 1994).

Este trabajo tiene como objetivo destacar la importancia ecológica de la Reserva Forestal El Montoso y registrar por primera vez para Panamá el género de Orthoptera: Tettigoniidae, Anaphidna Gorochov \& Cadena-Castañeda, 2012, dentro de la Reserva Forestal El Montoso.

\section{MATERIALES Y MÉTODOS}

Un espécimen de Anaphidna sp. prob. hernandezi (Figuras 3 A y B; 4 A y B) fue colectado en la localidad de la Reserva Forestal El Montoso, Las Minas, provincia de Herrera, Panamá; incluye dentro de sus límites corregimientos como Chepo, El Toro y Leones. Específicamente, el muestreo se realizó en las coordenadas 7운 $43^{\prime} 56.5^{\prime \prime} \mathrm{N}$ y $80^{\circ} 48^{\prime} 02.1$ " y a $600 \mathrm{msnm}$ (Figura 1). 


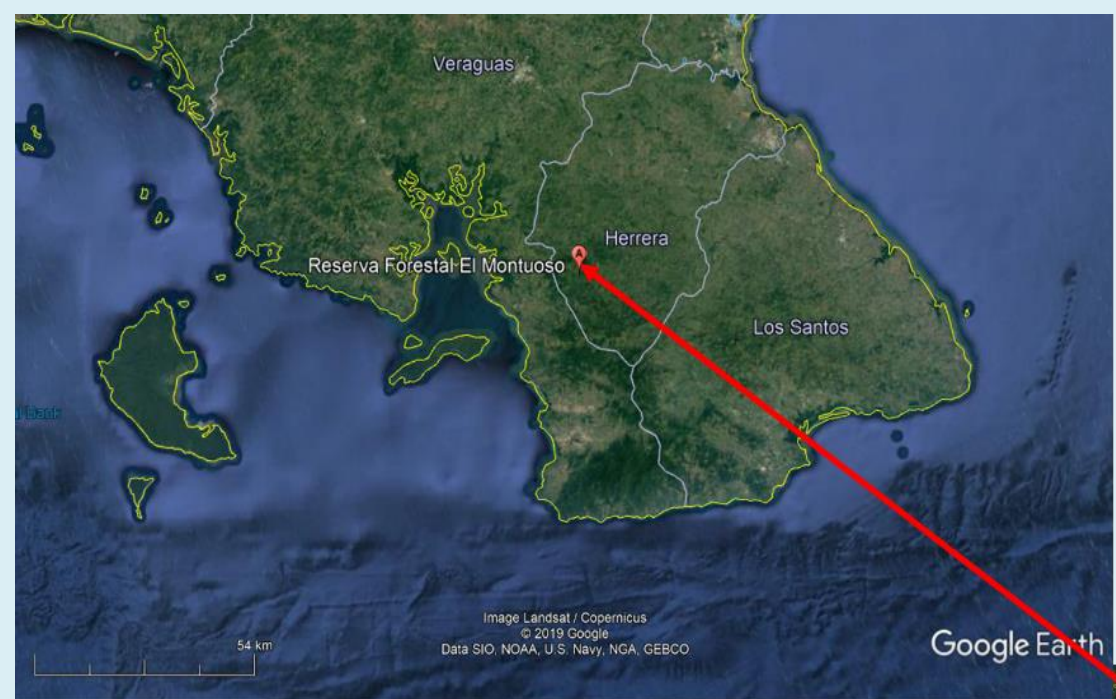

Figura 1. Mapa del sitio donde se localizaba Anaphidna sp. prob. hernandezi en Panamá.

La Reserva Forestal El Montoso es considerada como el último refugio de la diversidad en la provincia de Herrera, lo mismo que patrimonio natural de los panameños. Esta reserva fue creada con el objetivo de conservar y mantener, tanto la calidad del agua, como los caudales de los ríos circundantes. Además del cuidado de estas fuentes de agua, se pretende la protección de los bosques pluviales y el rescate de algunos humedales de la cuenca alta del río la Villa, que es el Rio más importante de la provincia de Herrera y abastece de agua a casi toda la población en la provincia.

En El Montoso sobresalen tres puntos altitudinales de relevancia: las cotas $987 \mathrm{msnm}$, $948 \mathrm{msnm} \mathrm{y}$ 939 msnm; esta última es llamada Loma El Montuoso. La segunda elevación montañosa en la región la representa la cordillera de La Huaca, con sus $745 \mathrm{msnm}$, unos $5 \mathrm{~km}$ al NEE de la cordillera de El Montoso. La temperatura media anual varía entre los $21-25^{\circ} \mathrm{C}$, siendo de $24.5^{\circ} \mathrm{C}$ para esta área; la evapotranspiración potencial anual es de unos 1565-1710 $\mathrm{mm}^{3}$. La temperatura máxima registrada para la región corresponde al mes de abril con $30.6^{\circ} \mathrm{C}$ y la mínima mensual se registró en el mes de enero con $23.7{ }^{\circ} \mathrm{C}$, del mismo año. Siendo la misma relativamente uniforme durante todo el año, alcanzando apenas una diferencia de $4{ }^{\circ} \mathrm{C}$ como promedio, entre las máximas y las mínimas (CODESA, 2003). El espécimen fue trasladado al Museo de Invertebrados G. B. Fairchild de la Universidad de Panamá donde se identificó el género utilizando el trabajo de Cadena-Castañeda et al. (2016), luego se almacenó y asignó el código MIUPOTCA002.

\section{RESULTADO Y DISCUSIÓN}

La Reserva Forestal El Montoso fue creada mediante la Ley $\mathrm{N}^{\circ} 12$ del 15 de marzo de 1977, con una extensión de 12,043 ha, y se ubicada en la provincia de Herrera, Distrito de Las Minas, en los Corregimientos de Chepo, El Toro y Leones. El Montoso se encuentra ubicada en el extremo Norte del macizo occidental de Azuero, región con la tasa de deforestación más elevada en todo el país; la reserva El Montoso está compuesta principalmente por ecosistemas de montaña; presenta una 
estructura de parches de bosques interconectados por diferentes sistemas de vegetación rivereña o bien bosques de galería y se estima que la vegetación nativa cubre unas 400 ha. Entre los ríos que nacen en esta reserva destacan La Villa, Mariato, Tebario y Suay.

En esta área fuertemente impactada, se puede ver fácilmente la fragilidad de los suelos y el avance de la frontera agrícola. Las actividades productivas del área incluyen la agricultura, la ganadería, la cacería y la extracción de leña para cocinar.

La Reserva Forestal El Montoso, es conocida como el "Pulmón de la provincia de Herrera", tiene un alto potencial de endemismo debido a su historia geológica (Froehlish \& Frohelish, 1987). En esta región se han identificado especies de flora endémica y amenazadas que se encuentran consideradas en convenios internacionales como CITES e UICN (Correa \& Valdespino, 1998).

En esta ocasión queremos seguir resaltando la importancia de la Reserva Forestal el Montoso al registrar por primera vez el género Anaphidna Gorochov \& Cadena-Castañeda, 2012 (Orthoptera: Tettigoniidae) para Panamá (Figuras 3 A, B y 4 A, B). Esta especie fue colectada a 600 msnm; llegó atraído a la luz de un foco $(100 \mathrm{~W})$ que se encuentra en la parte de afuera de la estación del Ministerio de Ambiente, ubicada en la Reserva Forestal El Montuoso, Las Minas Provincia de Herrera.

Cadena-Castañeda et al. (2016) elevan a rango de género a Anaphidna Gorochov \& Cadena-Castañeda, 2012. La especie Anaphidna rubricorpus Cadena-Castañeda, 2012 ha sido registrada para Costa Rica y Colombia, siendo muy probable su presencia en Panamá (Figura 2).

En las recientes publicaciones sobre la bioecología de los miembros de la subfamilia Phaneropterinae, a la que pertenece el género Paraphidnia, se ha determinado que es común que las especies Neotropicales presenten simpatria; por ejemplo, Gorochov (2012 a y b) pudo observar en la Amazonía que dos y hasta tres especies que viven en el dosel pueden vivir en el mismo arboles e incluso en la misma rama; el género Ceraia (tres especies), Euceraia (cuatro especies), Phylloptera (seis especies); datos similares fueron reportados por Nickle \& Castner, (1995) y Cadena-Castañeda \& Gorochov (2012).

Figura 2. Sitios y rango de distribución donde se han colectado $A$. rubricorpus (Costa Rica y Colombia) y A. prob. hernandezi (Panamá).

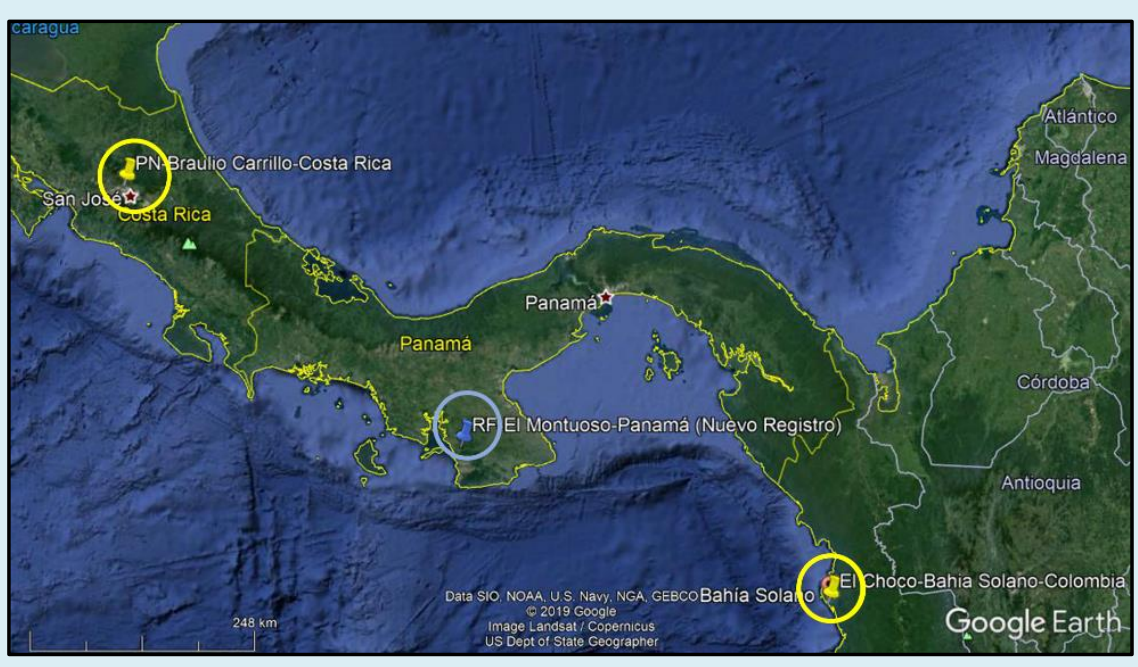

Se amplía el rango de distribución del género Anaphidna a nuestro país; es de esperarse que la especie Anaphidna rubricorpus esté en Panamá, ya que existe registro en Costa Rica, en el Parque Nacional Braulio Carrillo, y en Colombia en El Choco, Bahía Solano: Figura 2. 

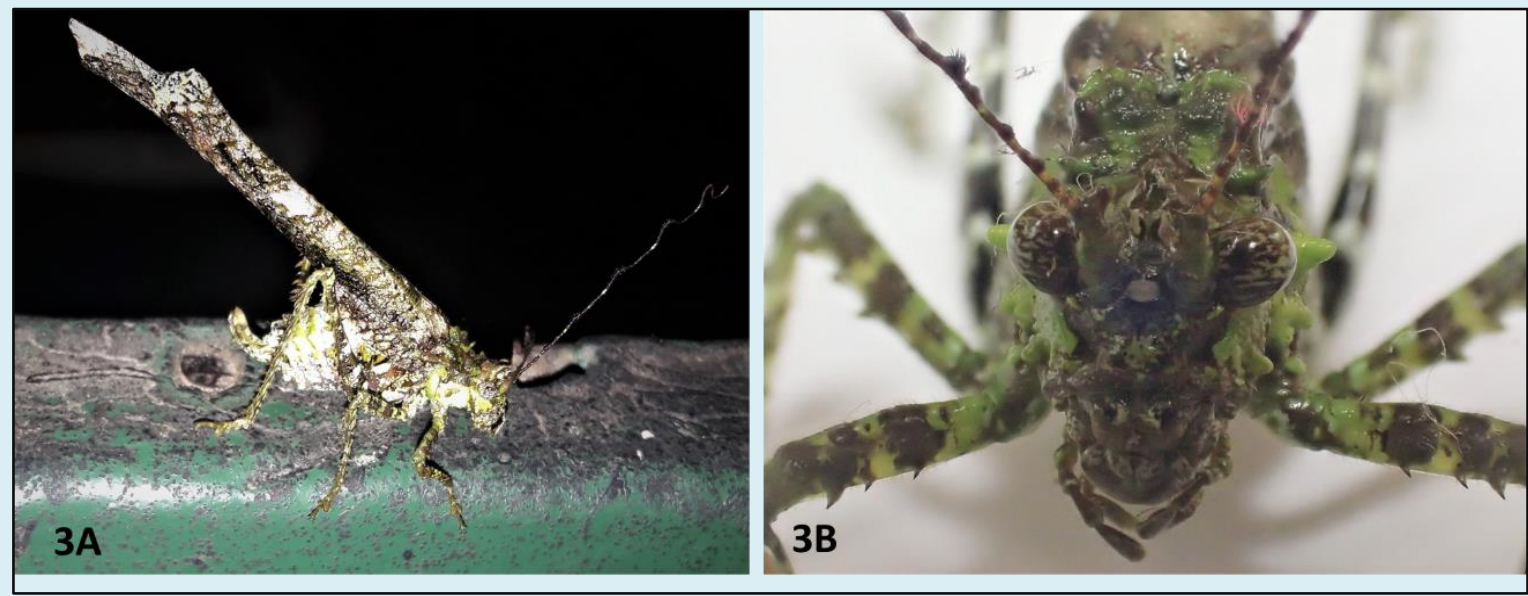

Figura 3. A. Vista lateral de Anaphidna sp. en el sitio de colecta. B. Vista de la cara frontal de la cabeza Anaphidna sp.
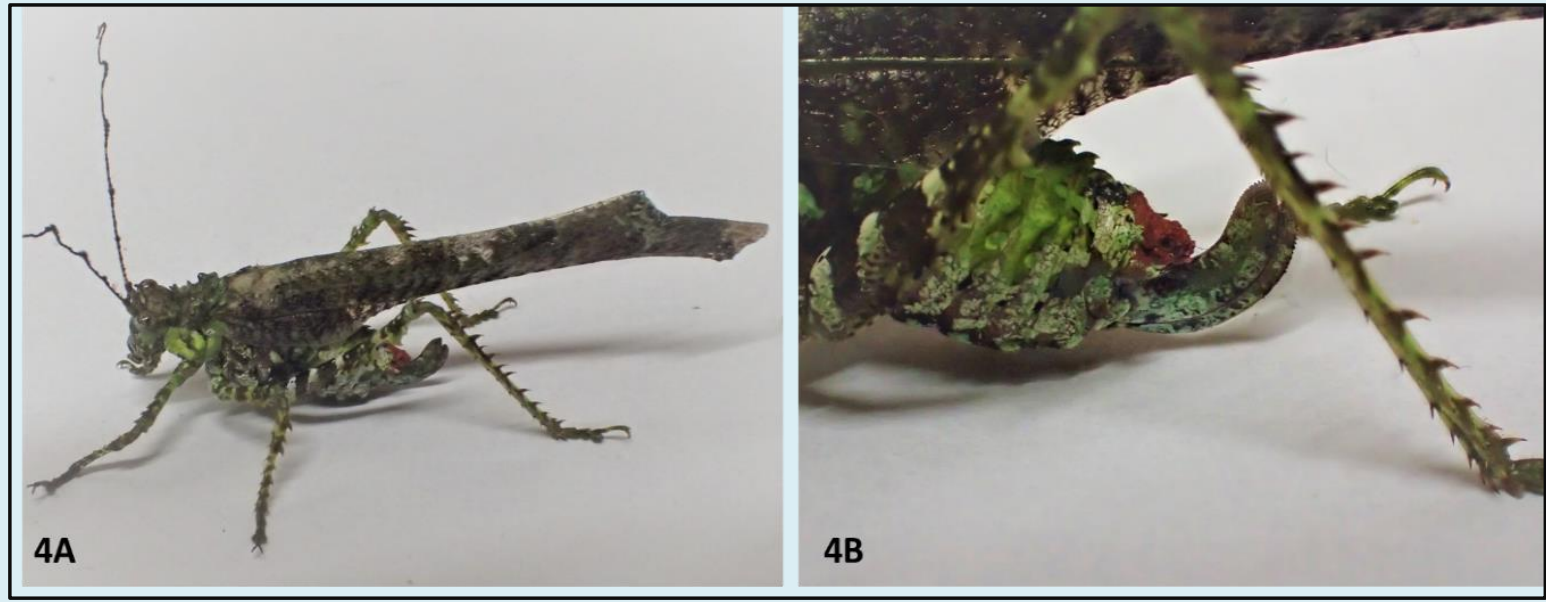

Figura 4. A. Vista lateral de Anaphidna sp. en el laboratorio. B. Vista lateral de los segmentos caudales de Anaphidna sp.

\section{AGRADECIMIENTOS}

Al personal del Ministerio de Ambiente en especial a la Regional de la provincia de Herrera, específicamente al personal de la Reserva Forestal El Montoso, por permitirnos el alojamiento, facilidades y el uso de instalaciones.

\section{REFERENCIAS}

CADENA-CASTAÑEDA, 0. \& GOROCHOV, A. 2012. Review of the Neotropical genus Paraphidnia (Orthoptera: Tettigoniidae: Phaneropterinae). Zoosystematica Rossica, 21(2): 204-233. 
CADENA-CASTAÑEDA, O.J., BRAUN, H. \& BUZZETTI, F.M. 2016. The tribe Dysoniini part V: The group Paraphidniae, with three new species from Guatemala and Ecuador (Orthoptera: Tettigoniidae: Phaneropterinae). Journal of Orthoptera Research, 25 (1): 15-23.

CODESA. 2003. Plan de Manejo de la Reserva Forestal El Montuoso. Autoridad Nacional del Ambiente, República de Panamá.

CORREA, M. \& VALDESPINO, I. 1998. Flora de Panamá, una de las más ricas y diversas del mundo. ANCON 5: 16-23. 28

EADES, DC., OTTE, D., CIGLIANO, MM., \& BRAUN, H. 2016. Orthoptera Species File Online. In. Vol. 2016. Version 5.0/5.0 edn.

FROHELICH, J \& FROHELICH, P. 1987. The status of Panama's endemic howling monkeys. Primate Conservation 8: 58 -62.

GIGLIO-TOS, E. 1898. Viaggio del Dr. Enrico Festa nella Republica dell'Ecuador et regioni vicine. VI. Ortotteri. Bollettino dei Musei di Zoologia ed Anatomia Comparata della R. Università di Torino. 13(311): 1-108.

GOROCHOV, A.V. 2012a. Systematics of the American katydids (Orthoptera: Tettigoniidae). Communication 1. Proceedings of the Zoological Institute RAS, 316(1): 3-21.

GOROCHOV, A.V. 2012b. Systematics of the American katydids (Orthoptera: Tettigoniidae). Communication 2. Proceedings of the Zoological Institute RAS, 316, 1, 3-21.

GWYNNE, DARRYL T.; BROWN, WILLIAM D. 1994. "La alimentación del compañero, la inversión de la progenie y las diferencias sexuales en los katydids (Orthoptera: Tettigoniidae)". Ecología Conductual.5 (3): 267 $-272$.

NICKLE, D.A. \& CASTNER J.L. 1995. Strategies Utilized by Katydids (Orthoptera: Tettigoniidae) against Diurnal Predators in Rainforests of Northeastern Peru. Journal of Orthoptera Research, 4: 75-88.

NICKLE DAVID, A. 1992. Katydids of Panamá (Orthoptera: Tettigoniidae). En: Insects of Panamá and Mesoamerica, Selected Studies. Diomedes Quintero and Annette Aiello. Oxford University Press, Oxford New York Tokyo.

REHN J. 1918. Description of one new genus and fifteen new species of tropical American Orthoptera. Transactions of the American Entomological Society, 44: 321-372, pl. 18-20.

RENTZ, D. 2010. " A guide to the katydids of Australia". Journal of Insect Conservation. 14 (6): 579 - 580.

ROBERTSON, L.; MEYER, J. 2010. Exploring Sound with Insects. Science Scope. 33 (5): 12.

SANTOS, A., AÑINO, Y. \& GÓMEZ, I. 2017. Primer registro de Celidophylla albimacula Saussure y Pictet, 1898 (Orthoptera: Tettigoniidae) en Panamá. Insecta Mundi 0597

Citar como: Santos, A., Cambra, R. \& Gómez, C. 2020. La Reserva Forestal El Montoso y el registro de la esperanza Anaphidna Gorochov \& Cadena-Castañeda 2012 (Orthoptera: Tettigoniidae) en Panamá. Mesoamericana 24(1): 22-27.

Anuncio importante: Este artículo cuenta con una versión anterior errada, la misma ha sido removida de la web, por lo que este anuncio busca evitar cualquier tipo de confusión. Este es el articulo original. 